\title{
Standoff Quantum Cascade Laser Photoacoustic Spectroscopy based Explosive Detection System
}

Ramesh C Sharma*, Gupta S, Deepak K, Sanchit G and Chandra H

Laser Science \& Technology Centre, DRDO, Delhi, India

\begin{abstract}
This paper focuses on the design and development of measurement technique and processing of signal for the detection of various explosive simulants like RDX(cyclo-trimethylene-tri-nitramine), TNT(Trinitro toluene), Sarin, TATP(Tri acetone triperoxide), their simulants like nitrobenzene, DNT(Dinitro toluene), DMMP(DiMethyl Methyl Phosphonate), acetone, propanol, etc. (in different states of matter) adsorbed on a metallic surface from a standoff distance ranging from few meters up to a distance of 25 meters in the wavelength range of 7-9 $\mu \mathrm{m}$. The focus also lies on the measurement methodologies and the instrumentation employed in these systems. A dedicated single screen, single user, user friendly Graphical User Interface(GUI) for controlling the entire system, acquisition and processing of the incoming signal and demonstration of results has been developed with the help of Laboratory Virtual Instrument Engineering Workbench (LABVIEW). The dual phase sensitive detection technique has been employed. The "Data Acquisition for Explosive Detection System" (DAEDS) also carries out precise operation sequencing, parameter control, parameter measurement and storage of data. The incoming signal profile has been normalized with respect to the reference laser profile to obtain the resultant graph. Various experiments have been conducted and the resultant graphs have been plotted with intensity on the $y$-axis and wave-number on the $x$-axis as shown in the results section of this paper. Furthermore, online determination of the explosive or the simulant has been carried out. An engineering proto-type system has been developed which indicates the detected explosive/ simulant using the developed software.
\end{abstract}

Keywords: DAEDS; Dual phase sensitive detection lock-in amplifier; Hazardous chemicals and Explosive detection system; Graphical User Interface; LABVIEW; Dual phase sensitive detection lock-in amplifier

\section{Introduction}

Terrorists are regularly using new techniques for mass destruction against civilian population. Therefore, the detection of explosives from safe standoff distances is very important for countering the terror attacks as it involves innocent civilian population. Keeping this in mind, governments all over the world are encouraging programs for safe standoff detection of explosives. This also enhances the economic growth of the nation. These threats include potential release of hazardous chemicals, biological warfare agents and explosives. In order to neutralize the impact of such threats, they require early detection from a safe standoff distance in the presence of ambient conditions. With the development of high power, miniaturized, tunable Quantum Cascade lasers (QCL), the Quartz Enhanced Laser Photoacoustic Spectroscopy (QE-LPAS) Technology has emerged as a powerful technique for standoff detection of explosives, chemical agents. This technique is an extension of conventional photo-acoustic spectroscopy technique (PAS) [1,2]. In this technique, QCL source is modulated at the resonant frequency of Quartz Crystal Tuning Fork (QCTF) (i.e. $32.8 \mathrm{KHz}$ ), which acts as a detector, and enables total insensitivity to ambient CW radiations. Details of the technique are explained in reference [1-3]. The main advantages of this technique is that it offers standoff detection of low concentration (up to ppb level) of chemicals/ explosives without using a sample collection step, by the detection of trace quantities of surface adsorbed chemicals/explosives [4-6].

The main aim was to develop a stand-alone, single user operable, single screen operable data acquisition system that could be sufficient is detecting the explosives from safe standoff distances. In the present paper we have outlined the methodology for the design and development of Data Acquisition for Explosive Detection System(DAEDS) which is applicable for the detection of explosive materials like RDX(cyclo-trimethylene-tri-nitramine), TNT(Trinitro toluene), Sarin, TATP(Tri acetone triperoxide) and their simulants like nitrobenzene, DNT(Dinitro toluene), DMMP(Di-Methyl Methyl Phosphonate), acetone, propanol, etc. (in different states of matter) adsorbed on a metallic surface from a standoff distance ranging from few meters up to a distance of 20 meters in the wavelength range of 7-9 $\mu \mathrm{m}$. This paper also reports the manner in which LABVIEW along with DAQ cards have been utilized by DAEDS to obtain critical parametric results and optimization of system parameters. This paper also presents Standoff QE-LPAS spectrum of explosive simulants/ molecules adsorbed at diffused aluminum plate from a distance of up to 20 meters in different states of matter. Experimentally, we have developed a Trolley mounted portable system for detection of explosives. In future with the increase in the power of QCL Laser due to technology advancement, the system will be upgraded to detect the Hazardous chemicals from safe distance up to $\sim$ hundreds of meters. The design of DAEDS is highly sophisticated, sensitive and compact. The mechanism and need of customized DAEDS is illustrated in the following paragraphs. LABVIEW is being utilized for the control of the equipments and processing of the incoming signal. Through LABVIEW all types of instrumentation equipments can be virtually configured without the need of actual hardware through a single screen GUI and via a single user.

*Corresponding author: Ramesh C Sharma, Laser Science \& Technolgy Centre DRDO, Delhi, 110054, India, Tel: 04024347630; E-mail: rameshsharma@lastec.drdo.in

Received August 26, 2014; Accepted October 31, 2014; Published November 10, 2014

Citation: Sharma RC, Gupta S, Deepak K, Sanchit G, Chandra H (2014) Standoff Quantum Cascade Laser Photoacoustic Spectroscopy based Explosive Detection System. J Laser Opt Photonics 1: 109. doi:10.4172/2469-410X.1000109

Copyright: (c) 2014 Sharma RC, et al. This is an open-access article distributed under the terms of the Creative Commons Attribution License, which permits unrestricted use, distribution, and reproduction in any medium, provided the original author and source are credited. 


\section{BLOCK DIAGRAM OF STAND OFF EXPLOSIVE DETECTION} TECHNOLOGY : LPAS SYSTEM

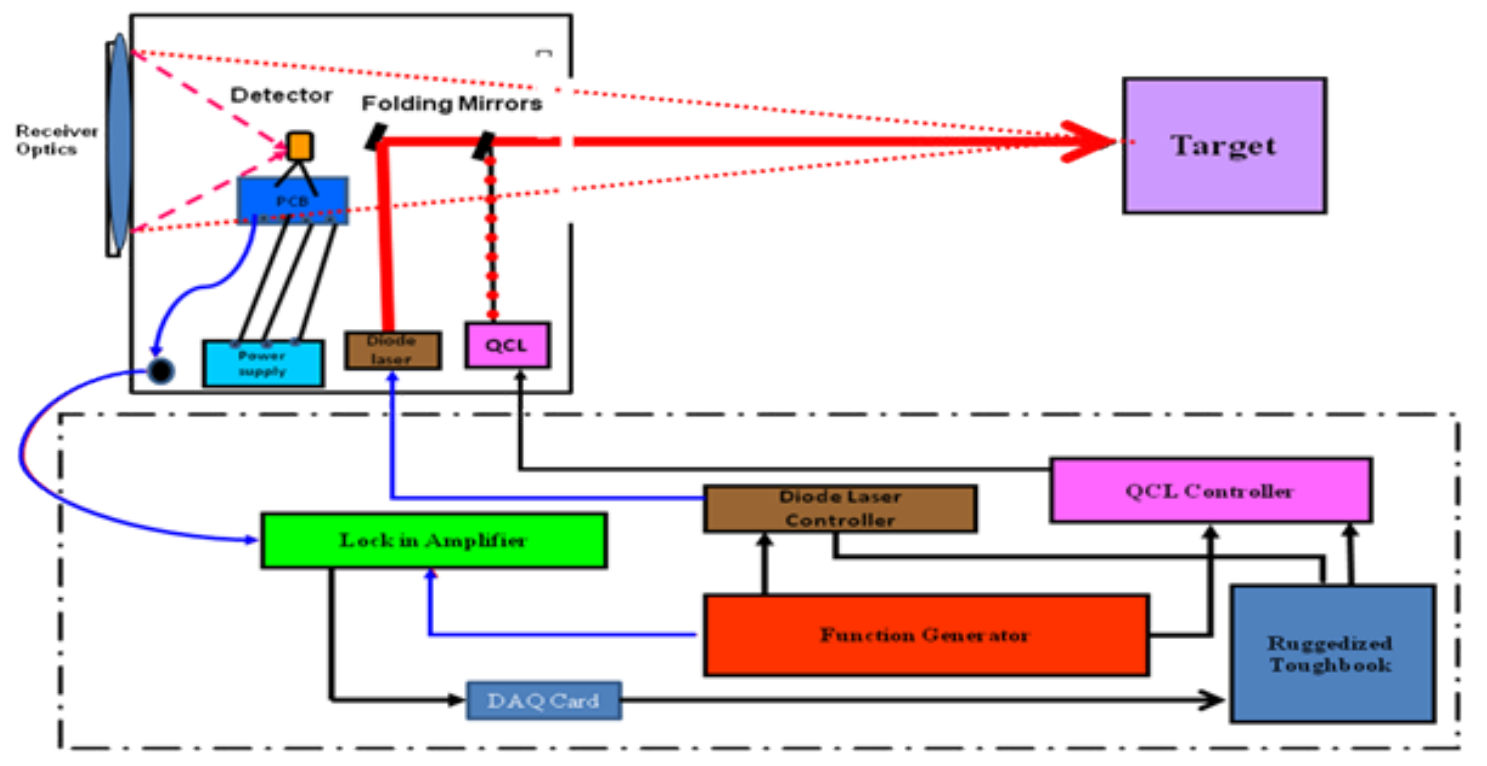

Figure 1: Block diagram of standoff explosive detection system.

1 mega ohm fixed in series with 1 mega ohm variable

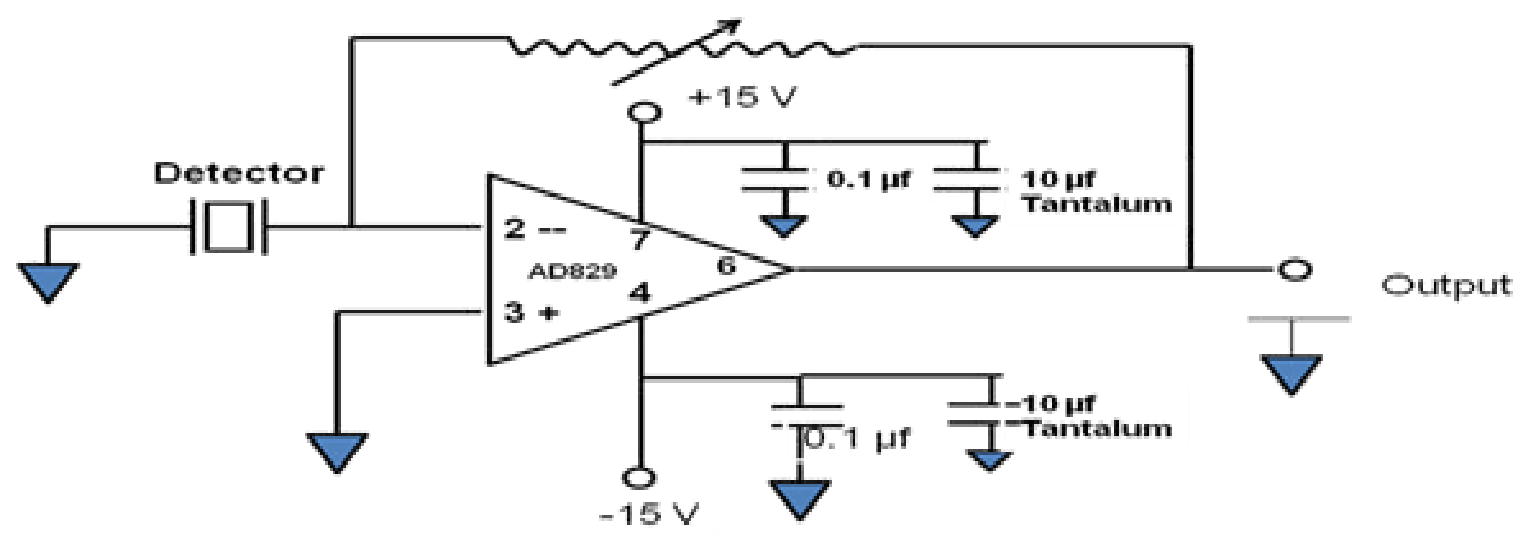

AD829 based Pre-amplifier circuit

Figure 2: AD829 based Pre-amplifier Circuit.

\section{Experimental}

System Design and Operation Figure 1 shows the block diagram of the standoff explosive detection system which shows the various electro-optical components that are required for successful detection of the explosives, chemicals, etc. In the following paragraphs different components are explained in detail shown in Figure 1.

\section{AD829 based pre-amplifier circuit}

The AD829 based pre-amplifier circuit is used for enhancing the amplitude of the incoming signal and converting the input current signal from the detector into voltage signal. This output signal can read by the lock-in amplifier. The pre-amplifier circuit consists of Quartz
Crystal Tuning Fork (QCTF) detector [3]. The detector is mounted on the PCB based pre-amplifier circuit shown in Figure 2. The QCTF detector converts the incident optical energy into electrical energy in the form of current. The resonant frequency of the QCTF based detector is of the order of $32.7 \mathrm{KHz}$ with bandwidth of $4 \mathrm{~Hz}$. As the current produced by the QCTF detector is of the order of nano amperes, hence High Speed, Low Noise Video Op Amp AD829 is employed. The AD829 is a low noise $(1.7 \mathrm{nV} / \sqrt{ } \mathrm{Hz})$, high speed op amp, has excellent gain, has a slew rate of $230 \mathrm{~V} / \mu$ s and settling time of $90 \mathrm{~ns}$ to $0.1 \%$, Input Offset Drift of $0.3 \mu \mathrm{V} /{ }^{\circ} \mathrm{C}$. Moreover, the feedback resistance has been taken to be variable so that gain of the pre-amplifier can be changed as and when required. Because of the above features of the AD829 at the 
Citation: Sharma RC, Gupta S, Deepak K, Sanchit G, Chandra H (2014) Standoff Quantum Cascade Laser Photoacoustic Spectroscopy based Explosive Detection System. J Laser Opt Photonics 1: 109. doi:10.4172/2469-410X.1000109

Page 3 of 8

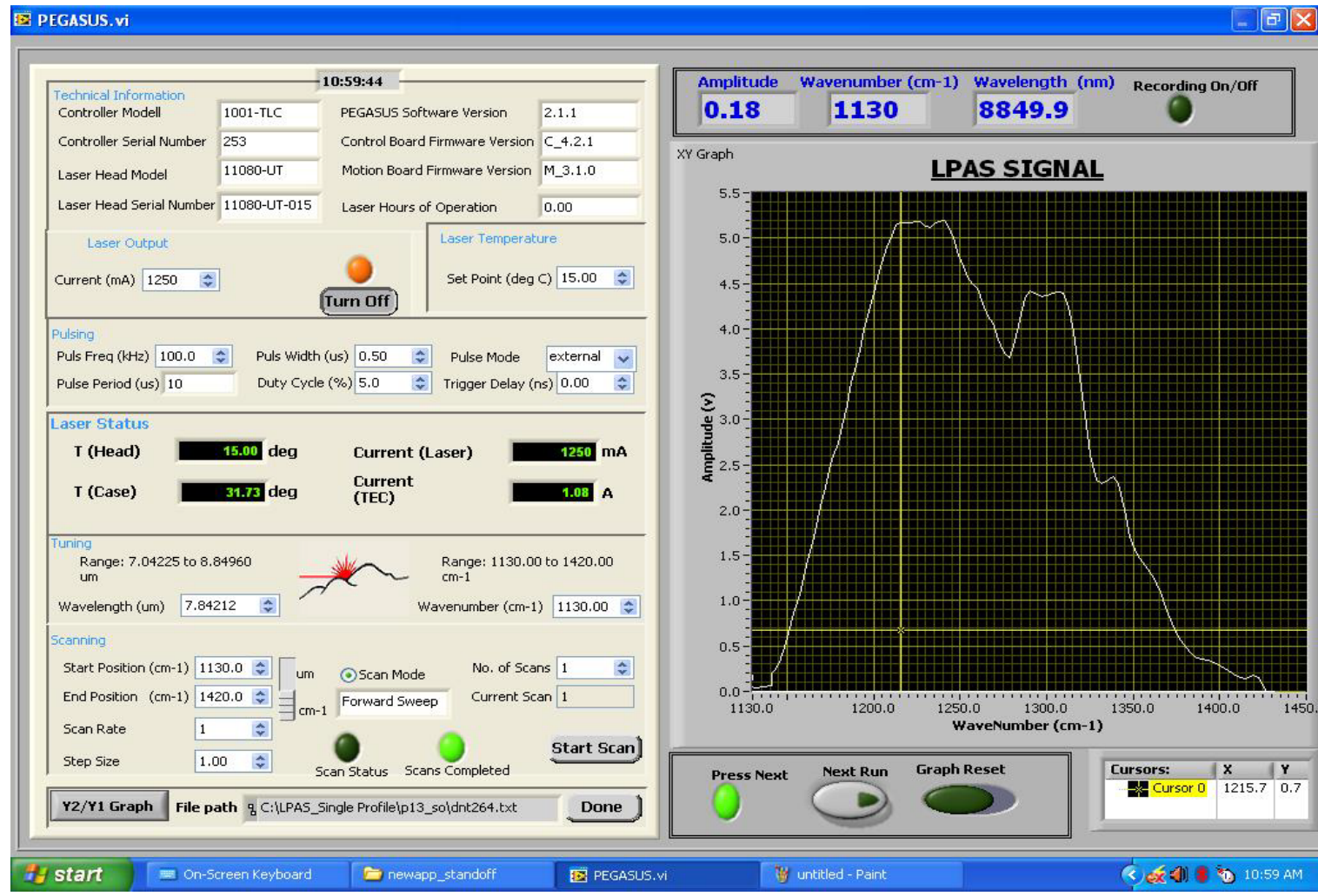

Figure 3: Single screen GUI.

frequency of interest, AD829 based pre-amplifier was fabricated in the trans-impedance mode as shown in Figure 2.

Tunable laser: The Tunable Laser System consists of a Laser Controller, a tunable Laser Head, and an umbilical cable assembly [7]. The purpose of the laser system is to provide tunable, mid-IR, pulsed laser radiation over a specified tuning range. The system provides laser Tunability, temperature control, and laser current control. The laser controller is interfaced with the Computer through any one of the Controller's data ports viz. USB port, GPIB port or RS232 port. Upon power-up, the Controller automatically interrogates the Laser Head to determine its center frequency (tuning range), and other Laser Head specific information. All this information is returned by the laser to the controller which in turn can be viewed on to the computer [8]. All the parameters of the laser viz. laser scan rate, scan range, number of scans, step size can be controlled through a standard GPIB cable, which in turn is connected to the computer.

Function generator (Tektronix AFG3102): In the experiment, dual channel function generator [9] is used to modulate the laser and also used by the lock-in amplifier as a reference signal, to lock on to the incoming signal from the PCB based pre-amplifier. It plays a very critical role in the experiment. The most important criteria for the lockin amplifier to lock is that the frequency of both the channels of the dual channel function generator should be same as that of the resonant frequency of the QCTF based detector viz. 32.7KHz.

Lock-in Amplifier (SR830): A lock-in amplifier use a technique known as dual phase-sensitive detection to single out the component of the signal at a specific reference frequency and phase and thus eliminates the phase dependency of the output signal of the lock-in [10]. The dual phase sensitive technique enables the lock-in amplifier to be totally independent of the phase of the incoming signal. The lock-in amplifier locks the incoming signal with the reference frequency signal and rejects all the other noise frequencies.

\section{Data acquisition for explosive detection/hazardous chemicals system (daeds)/daq}

A general purpose data acquisition system for explosive detection system (DAEDS) has been designed and developed to meet the realtime requirements of operational control, acquisition, measurement, monitoring, display, storage, and analysis of parameters of QCL and various explosives/chemical materials. The data acquisition system successfully implements the requirements of sequential and switching control of laser output, the online adjustment and display of parameters such as laser temperature, laser wave-number, laser current, laser scan rate, laser scan start and end position and storage of these parameters. The DAEDS successfully implements the diagnostics, measurement of incoming signal intensity and online detection of explosive/chemical present. A user friendly, single screen, single user operable GUI (as shown in Figure 3) was conceptualized and developed using LABVIEW which is used for operating the entire system [11]. The PC-based approach is selected because of the advantages of functionality and flexibility. The main features of the DAEDS are as follows in Figure 3.

1) The setting up of initial laser parameters and their finalization. The following laser parameters can be set as per the requirement:- 
Citation: Sharma RC, Gupta S, Deepak K, Sanchit G, Chandra H (2014) Standoff Quantum Cascade Laser Photoacoustic Spectroscopy based Explosive Detection System. J Laser Opt Photonics 1: 109. doi:10.4172/2469-410X.1000109

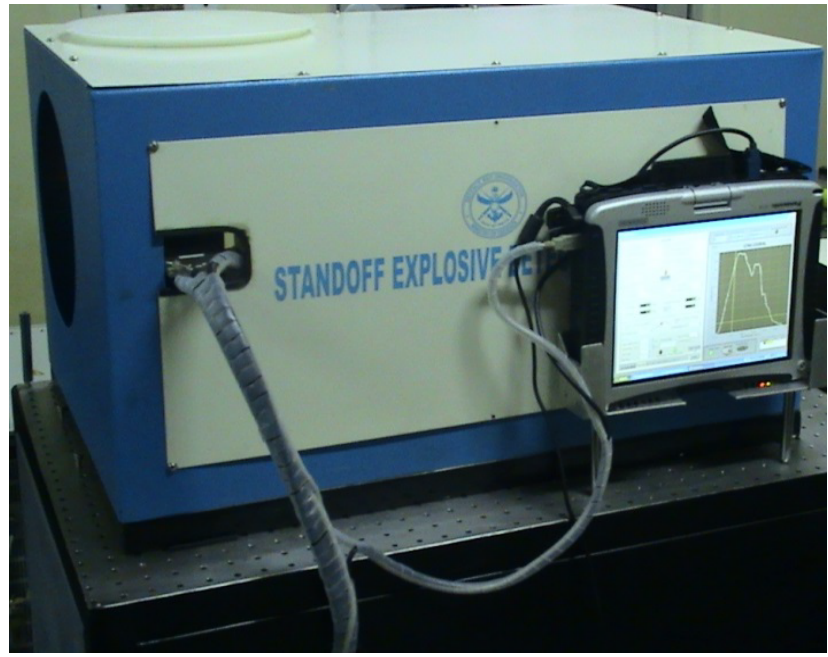

Figure 4: Trolley Mounted Prototype System.

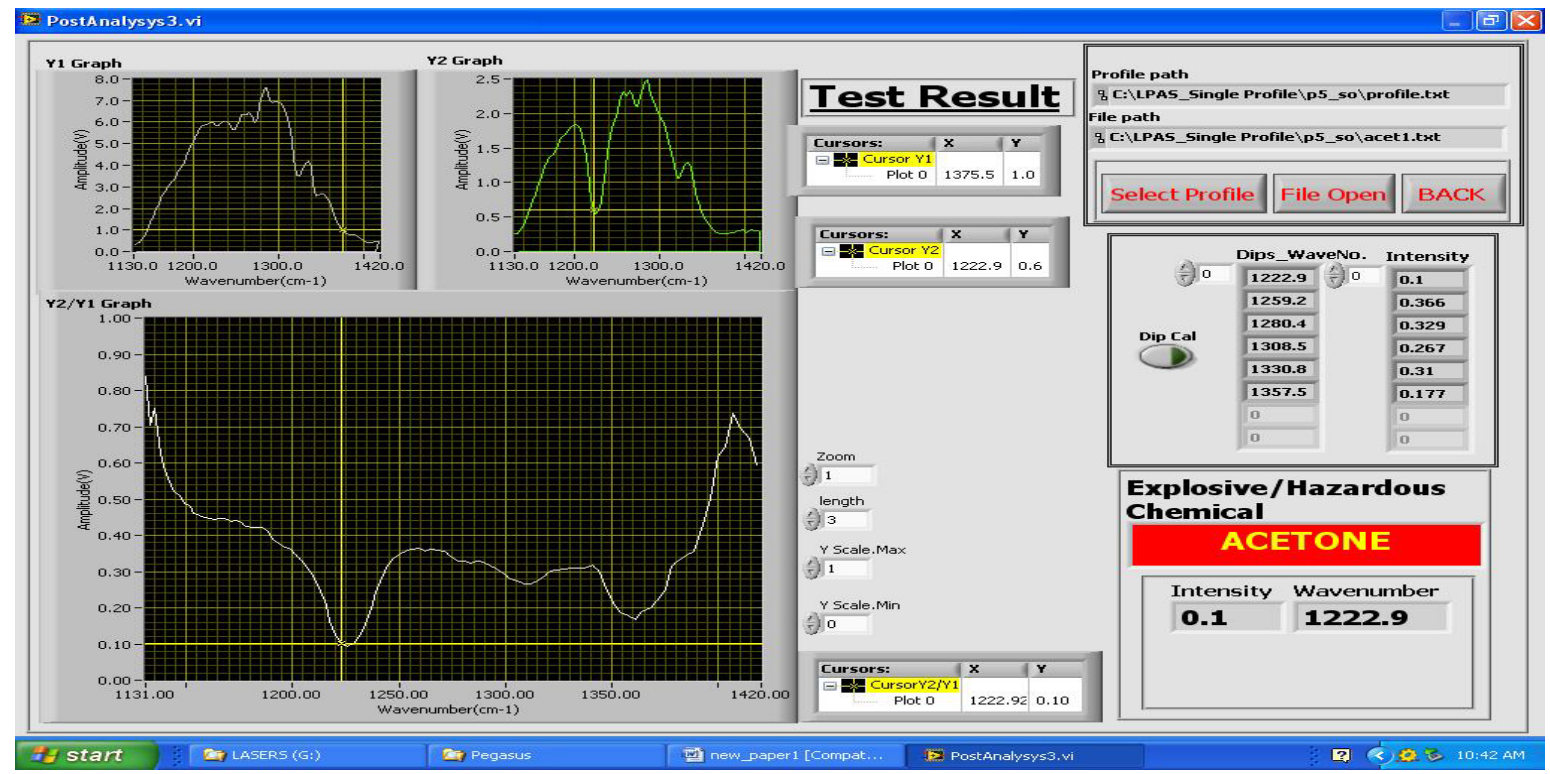

Figure 5a: Test Result GUI showing detection of acetone in liquid form from diffused aluminium surface.

a) Laser Current

b) Laser Pulse mode = internal/external (internal- used for alignment purposes, external- It is used when laser is modulated by a pulse waveform generated by function generator module for the detection of explosives)

c) Laser wave number $=$ as per the frequency range of the laser head

d) Laser Scan settings: Start position, Stop position, Scan rate, Step size, Scan Mode(Forward Sweep, Automatic Scan, Forward and Reverse Sweep), number of scans to be done

2) After setting the laser parameters, the laser is sent to the target. The laser is interfaced with the computer through laser controller
3) The graph on the main screen shows the laser profile on the target. A provision of the "Next Run" as shown in the GUI is made if two or more graphs are required to be seen on the same screen. This helps in comparison of two or more graphs

4) All the profiles viz. laser profile, explosive profile are stored separately in a file. They can be compared and after comparison the type of the explosive can be detected

5) A library of various explosive has been made and stored in the computer through which the profiles of the different explosives/ chemical agents can be compared and then detected

6) A multifunction Data Acquisition Card USB 4716 has been utilized for measuring the incoming signal [12]. This multifunction card is USB compatible and can be connected to the computer through a USB cable. It also offers the advantage 
Citation: Sharma RC, Gupta S, Deepak K, Sanchit G, Chandra H (2014) Standoff Quantum Cascade Laser Photoacoustic Spectroscopy based Explosive Detection System. J Laser Opt Photonics 1: 109. doi:10.4172/2469-410X.1000109

Page 5 of 8

of having digital input channels, digital output channels and analog output channels. It offers 16 single-ended/ 8 differential analog input channels with 16-bit resolution, $0.15 \%$ accuracy at full scale, up to $200 \mathrm{kS} / \mathrm{s}$ throughput, over voltage protection, 16 digital I/O lines and 1 user counter, and two 16-bit analog outputs. It obtains all required power from the USB port, so no external power connection is ever required

7) A trolley mounted prototype system has been developed as shown in Figure 4 in which all the opto-electronic components including the laser, pre-amplifier, the DAQ cards, etc. are mounted inside the metallic enclosure. This trolley mounted prototype system can be taken to any place for testing of the explosives. All the operation of the entire system can be conducted through a single screen GUI shown in Figure 4.

\section{Results and Discussions}

This section deals with the results and the necessary discussions. Various graphs viz. laser profile, explosive profiles for the detection of Acetone, Acetone in vapor form, Propanol, DNT, DMMP and nitrobenzene are shown in Figure 5a-5f. Initially, the laser profile was recorded and then in the second run the explosive/chemical profile was recorded. The laser profile has to be recorded only once at the starting of the experiment. Very frequent changes in the ambient atmospheric conditions in the near earth environment causes the laser profile to change. Therefore, it is compulsory to record laser profile every time we start an experiment. This recorded laser profile can be utilized for detection of different explosives. The laser profile graph has been denoted as Y1 graph and the explosive profile graph has been denoted as $\mathrm{Y} 2$ graph. Both these profiles were recorded and processed to obtain a software compatible profile for the detection of dips in efficient way. This normalizes the explosive profile with reference laser profile to obtain the resultant graph and hence clearly indicates the dips that are generated because of the presence of explosive. The target was kept at a distance of $20 \mathrm{~m}$ from the trolley mounted system. The explosives/ chemicals have been detected in all the three states of matter. The data obtained indicates repeatability. The detection of hazardous materials has been performed by using both the online detection and the post processing methods. The false alarm rate has been found out to be approximately $5 \%$ on the detected explosive materials. The low false alarm rate indicates the very high accuracy of the developed data acquisition system. Moreover, the response time of the system is of the order of few milli seconds. The data of the online detection was stored in various files for post detection analysis. The dip position of the various explosive materials depends on various factors like atmospheric conditions, distance of the target from the sensor, characteristics of the explosive material. Therefore, the dip values lie within a certain band of $\pm 5\left(\mathrm{~cm}^{-1}\right)$ from a central value. Therefore, there is a slight change in the value of dip positions every time an experiment is conducted. The software has been designed in such a way that a slight change of \pm 5 $\left(\mathrm{cm}^{-1}\right)$ in the wave-number has been accounted for. Moreover, a 'zoom' factor has been added in all the graphs for increasing the amplitude in all the graphs. The total time taken for processing the information and for detection of hazard and displaying the hazard on the screen is less than $5 \sec$ (Figure 5).

The LPAS signal is very high SNR at 20 meter distance. In further experiments standoff LPAS will be demonstrated up to 50 meter. Sensitivity also will be improve using the Laser power with modulated frequency of the QCTF detector.

\section{Conclusion}

The DAQ system has been able to successfully demonstrate the detection of stimulants of explosives. The gain of the AD829 based pre-amplifier circuit can be modified just by changing the value of the variable feedback resistance. In this way the output of the pre-amplifier

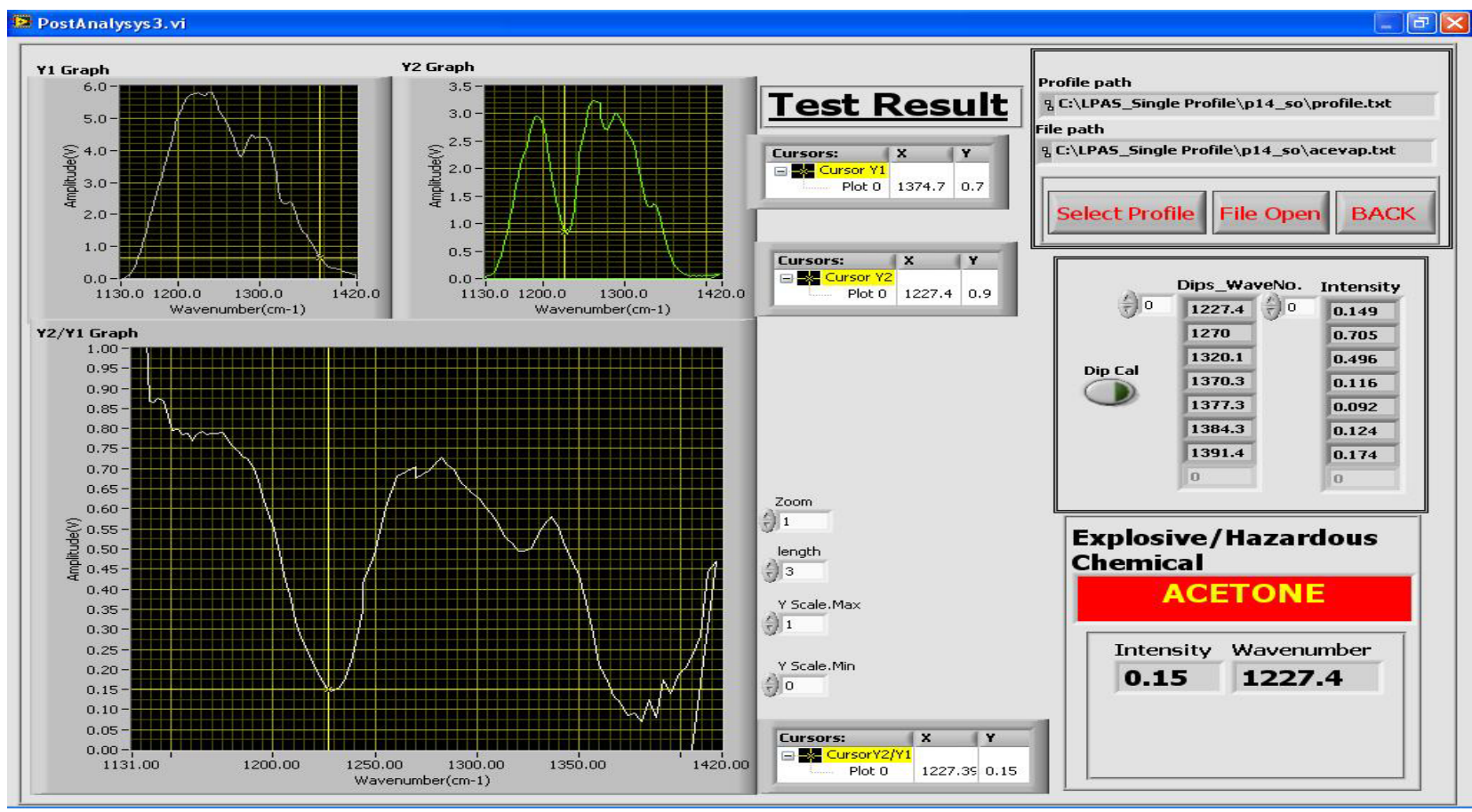

Figure 5b: Test Result GUI showing detection of acetone in vapour phase. 
Citation: Sharma RC, Gupta S, Deepak K, Sanchit G, Chandra H (2014) Standoff Quantum Cascade Laser Photoacoustic Spectroscopy based Explosive Detection System. J Laser Opt Photonics 1: 109. doi:10.4172/2469-410X.1000109

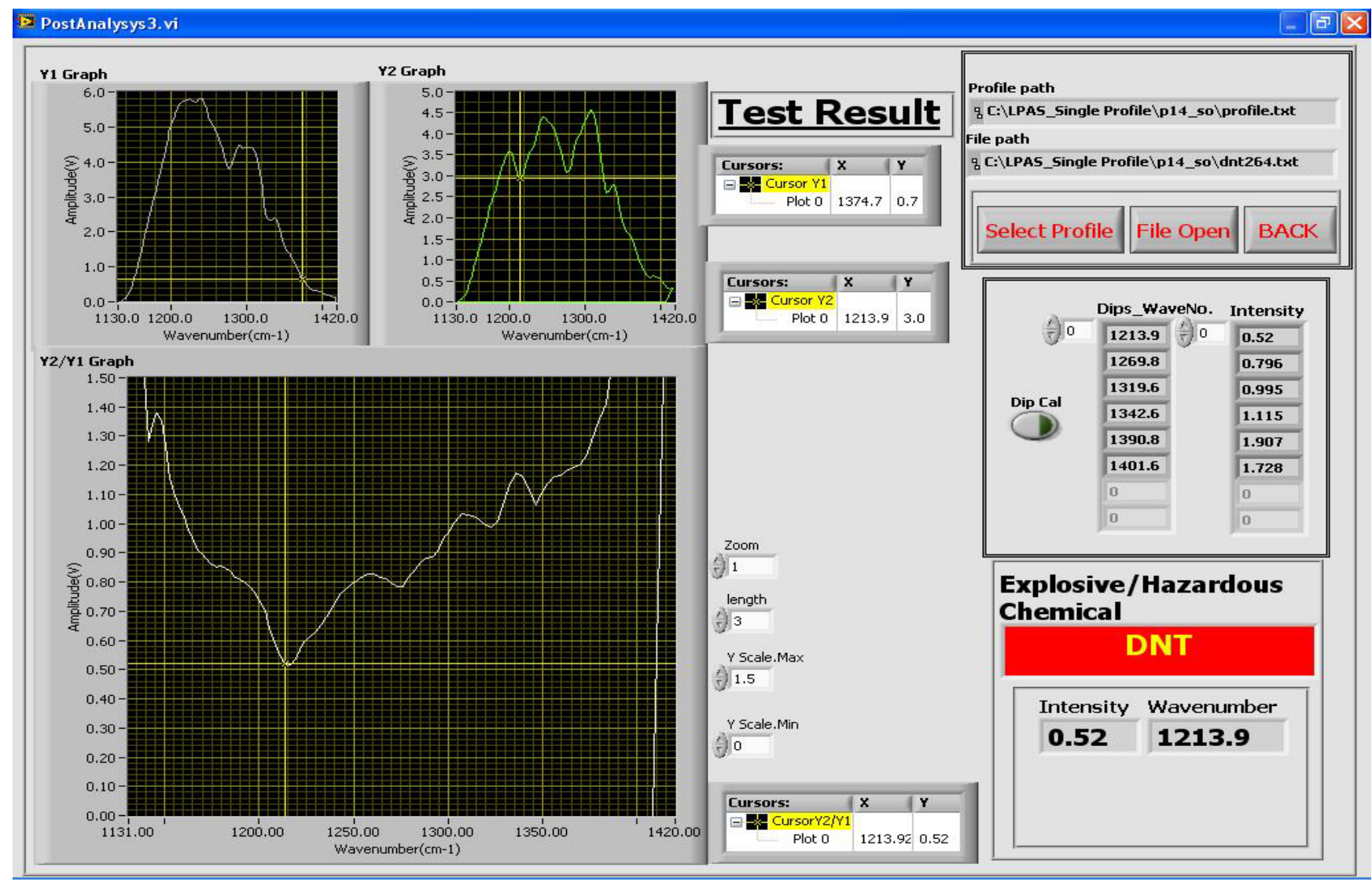

Figure 5c: Test Result GUI showing detection of DNT power adsorbed on diffused aluminum surface.

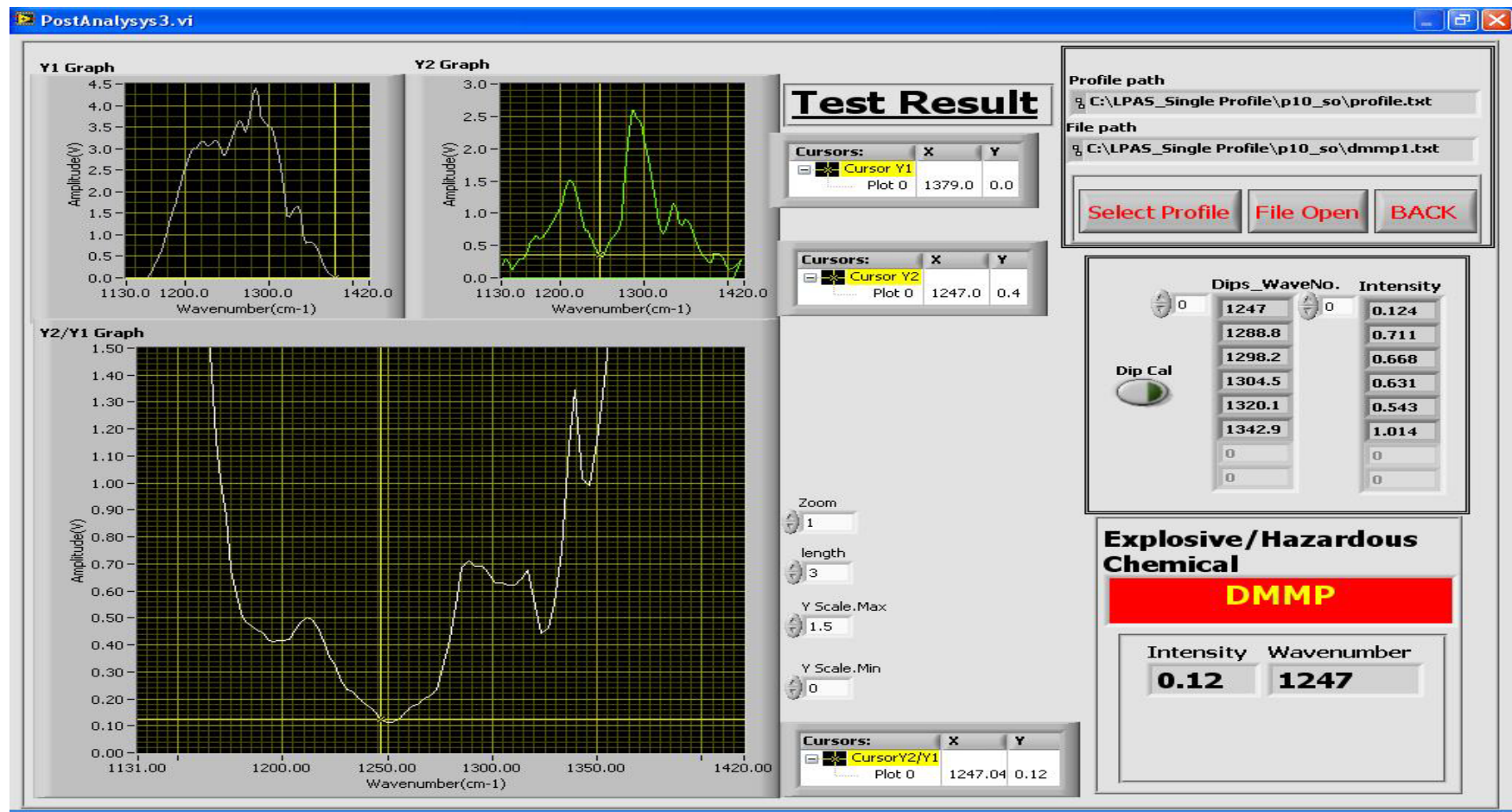

Figure 5d: Test Result GUI showing detection of DMMP in liquid phase on diffused aluminum surface. 
Citation: Sharma RC, Gupta S, Deepak K, Sanchit G, Chandra H (2014) Standoff Quantum Cascade Laser Photoacoustic Spectroscopy based Explosive Detection System. J Laser Opt Photonics 1: 109. doi:10.4172/2469-410X.1000109

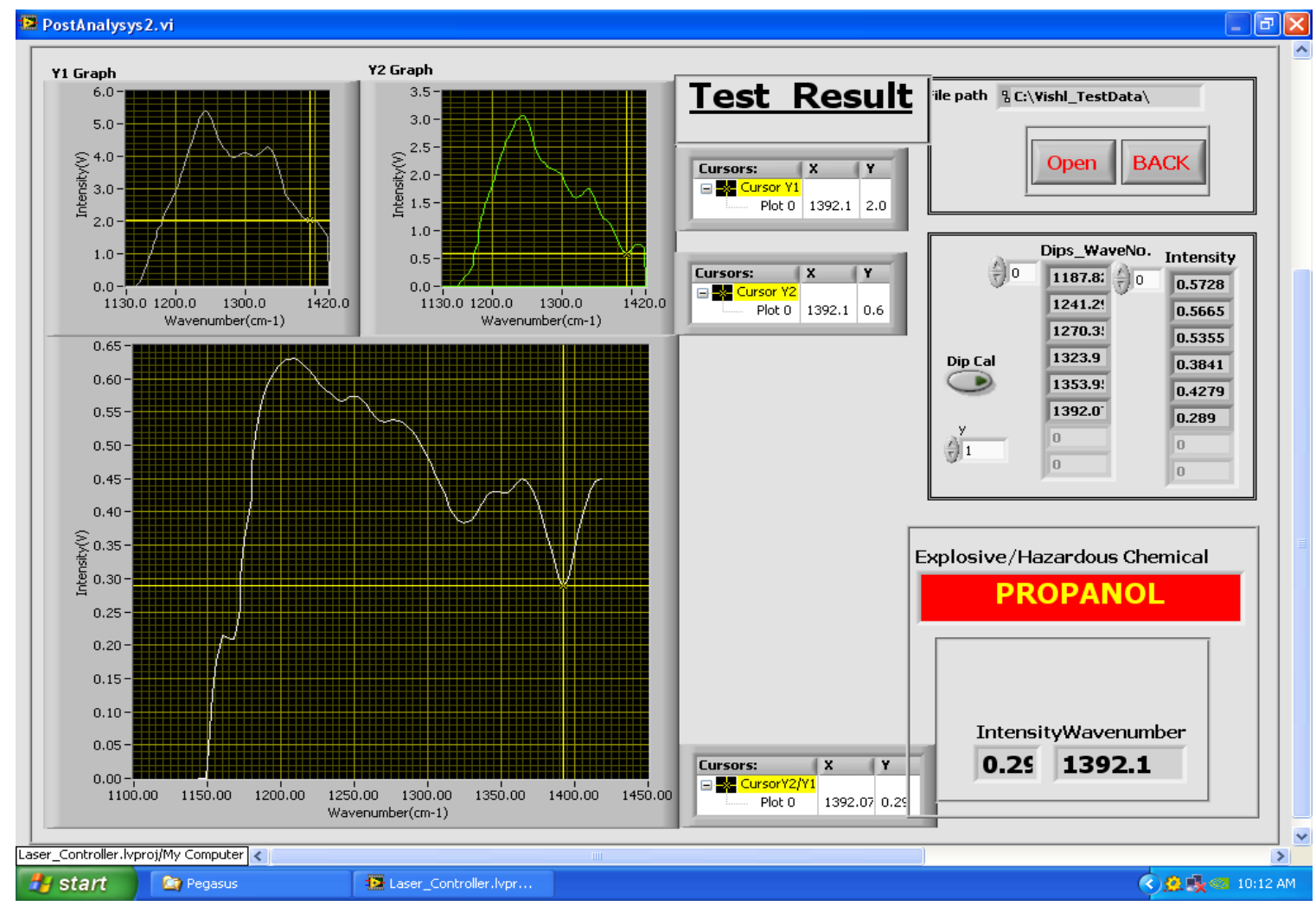

Figure 5e: Test Result GUI showing detection of propanol in liquid form from diffused aluminium surface.

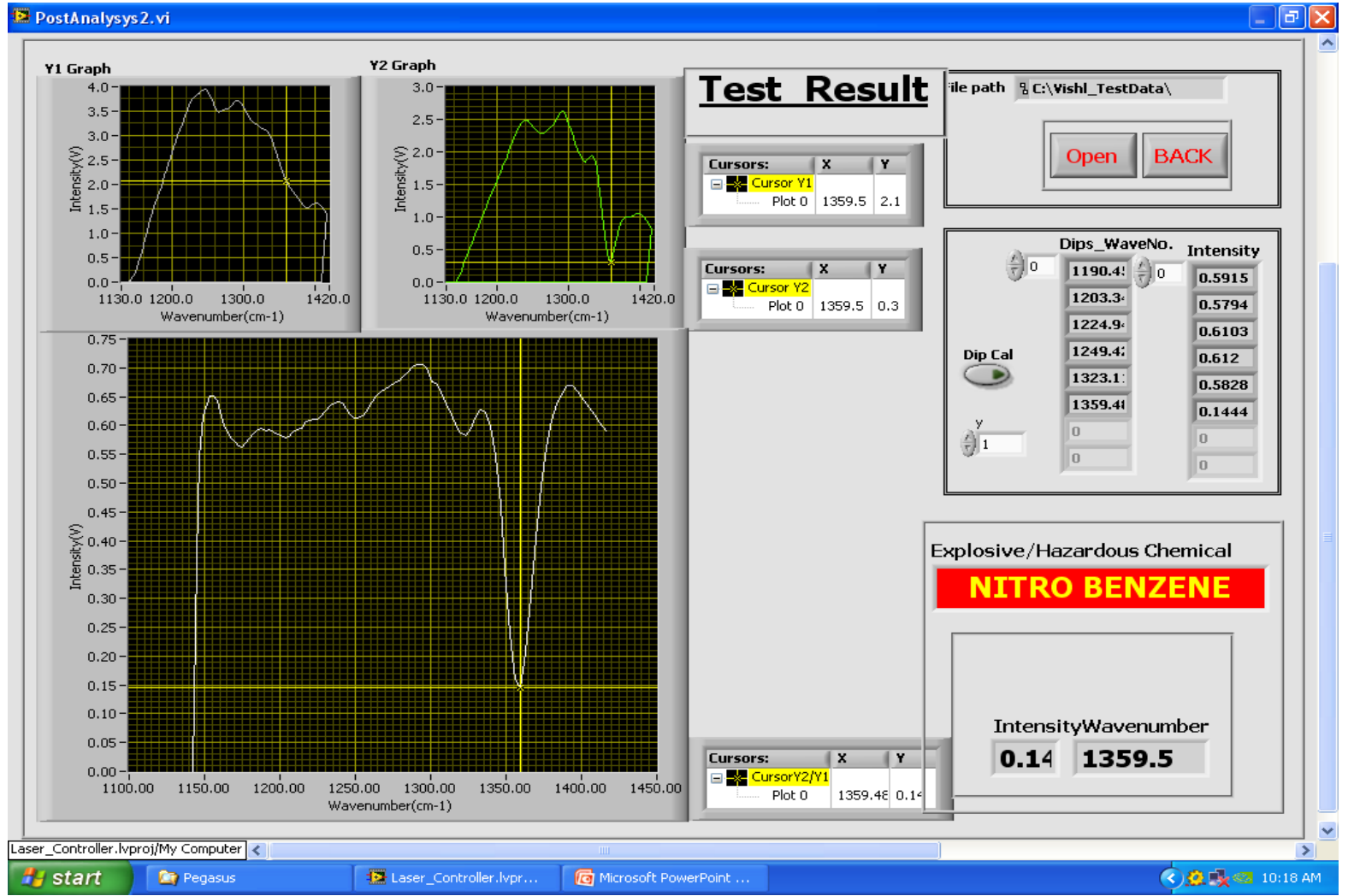

Figure 5f: Test Result GUI showing detection of Nitrobenzene in liquid form from diffused aluminium surface. 
Citation: Sharma RC, Gupta S, Deepak K, Sanchit G, Chandra H (2014) Standoff Quantum Cascade Laser Photoacoustic Spectroscopy based Explosive Detection System. J Laser Opt Photonics 1: 109. doi:10.4172/2469-410X.1000109

Page 8 of 8

can be changed as per the intensity of the incoming signal. The software has been designed in such a way that a change in the value of dip position within a particular range does not affect the detection of the explosive materials. These ranges are user defined and are different for different explosive materials. The lock-in amplifier, function generator, Quantum Cascade Laser etc. are controlled through the data acquisition system. Moreover, whole system is user friendly and controlled through a single screen GUI and by a single user.

We have successfully developed a trolley mounted movable engineering prototype system which is capable of detecting explosives/ chemicals in trace amounts without using sample collection step and from safe standoff distances. This system is capable of detecting the explosives/chemicals with concentration of the order of $100 \mathrm{nl}$ in liquid, $1.0 \mu \mathrm{g} / \mathrm{cm}^{2}$ explosive/ simulants at adsorbed surface and $1.0 \mathrm{ppm}$ in vapor phase from a safe standoff distance ranging from few meters up to 20 meter. The experimentally determined absorbed wavelengths of above mentioned explosives/chemicals are matching with the absorbed wavelengths as provided in the literature for the respective explosives/ chemicals. This confirms the accuracy of our system. Moreover, the system is easy to use as it has a single screen GUI which is single user operable. Presently, we are working on miniaturization and automation of this system for development of a Tripod mounted system having increased sensitivity, selectivity and target ranges.

\section{Acknowledgement}

Authors are thankful to discuss of the present with Professors Thomas Thundat, Alberta University, Canada, S N Thakur, BHU, J P Singh Mississippi State University, USA

\section{References}

1. Van Neste CW, Senesac LR, Thundat T (2008) "Standoff photoacoustic spectroscopy" Applied Physics Letters 92234102.

2. Gurton K, Felton M, Tober R(2009) ARL-TR-4782, US Army Research Lab.

3. Sharma RC, Kumar D, Bhardwaj N, Chandra H, Maini A K (2013) "Portable detection system for standoff sensing of explosives and hazardous materials". Optics Communication 309: 44-49

4. Van Neste CW, Senesac LR, Thundat T (2009) "Standoff Spectroscopy of Surface Adsorbed Chemicals”. Analytical Chemistry 81: 1952-1956.

5. Pohlkötter A, Willer U, Bauer C, Schade W (2009) "Resonant tuning fork detector for electromagnetic radiation". Applied Optics 48: 119-125.

6. Barbic M, Eliason L, Ranshaw J (2007) "Femto-Newton force sensitivity quartz tuning fork sensor". Sensors and Actuators A 136: 564-566.

7. AD829 High Speed, Low Noise Video Op Amp Data Sheet (2014)

8. D11-00002-01 REV A Tunable laser system user manual.

9. D09-00002-01 REV A User Commands Mid-IR External cavity Laser System.

10. MODEL SR830 DSP Lock-In Amplifier Manual Rev 2.5 (2011).

11. LABVIEW user manual (1998).

12. Multifunction Data Acquisition Card USB 4716 user manual (2014). 